\title{
Pilot study investigating the prognostic significance of thymidine phosphorylase expression in patients with metastatic breast cancer: a single institution retrospective analysis
}

\author{
This article was published in the following Dove Press journal: \\ OncoTargets and Therapy \\ 21 April 2015 \\ Number of times this article has been viewed
}

\author{
Anna Lisa Tedeschi' \\ Zohreh Eslami ${ }^{2}$ \\ Evgenia Garoufalis' \\ Ramy R Saleh ${ }^{3}$ \\ Atilla Omeroglu ${ }^{2}$ \\ Gulbeyaz Altinel ${ }^{2}$ \\ Maria Ait-Tihyaty ${ }^{4}$ \\ Bertrand Jean-Claude ${ }^{4}$ \\ Catalin Mihalcioiu' \\ 'Division of Medical Oncology, \\ Department of Medicine, McGill \\ University Health Center, Royal \\ Victoria Hospital, ${ }^{2}$ Department of \\ Pathology, ${ }^{3}$ Department of Medicine, \\ McGill University, ${ }^{4}$ Cancer Drug \\ Research Laboratory, Department \\ of Medicine, McGill University Health \\ Center, Royal Victoria Hospital, \\ Montreal, QC, Canada
}

Background: The thymidine phosphorylase (TP) enzyme is expressed in higher levels in cancer tissue when compared with normal tissue. It is involved in the intratumoral activation of widely prescribed pyrimidine-derived antimetabolites such as $5^{\prime}$-deoxy-5-fluorouridine and capecitabine $\left(\mathrm{Xeloda}^{\circledR}\right)$. The purpose of this study was to determine the clinical correlation between TP expression in tumor tissue and the clinical outcome of capecitabine-based therapy in patients with locally advanced (stage III) or metastatic breast cancer (stage IV).

Methods: The following variables were analyzed as potential determinants of benefit from a capecitabine-based therapy: TP expression, estrogen receptor(ER) and progesterone receptor (PR) status, human epidermal growth factor receptor-2 status, and Ki67 status. This was accomplished by immunohistochemical analysis of paraffin-embedded cancer tissues from 18 patients with breast cancer treated with at least one cycle of capecitabine. Clinical outcome was measured as time to progression.

Results: TP staining intensities in both the invasive and in situ components in patients with lobular and ductal carcinomas were reported. Higher levels of TP in the invasive component were expressed in ER-negative tumors when compared with ER-positive tumors $(P<0.05)$. The ER-positive group expressing lower levels of TP had a median time to progression of 13 months compared with the ER-negative group expressing higher levels of TP which had a median time to progression of 7.5 months $(P=0.14)$.

Conclusion: Patients with ER-positive tumors expressing lower levels of TP exhibit a longer time to progression when compared with patients with ER-negative tumors. Consequently, tumor TP expression does not seem to predict the outcome of capecitabine-based chemotherapy.

Keywords: thymidine phosphorylase, metastatic breast cancer, prognostic significance

\section{Introduction}

According to the Canadian cancer statistics for 2013, breast cancer (BC) is the most common cancer and the second leading cause of death from cancer among Canadian women. It is estimated that 23,800 women in Canada will be diagnosed with $\mathrm{BC}$, representing $26 \%$ of all new BC cases in women in $2013 .{ }^{1}$ Approximately $6 \%-10 \%$ of patients with $\mathrm{BC}$ have metastatic disease at the time of initial diagnosis, and $20 \%-30 \%$ of patients diagnosed with early-stage BC will eventually develop recurrent or metastatic disease. ${ }^{2,3}$ Metastatic breast cancer (MBC) remains incurable, and its management is palliative despite the extensive research in this field and emergence of novel therapies. In $90 \%$ of MBC patients, treatment failure is attributed to resistance to chemotherapeutic agents. ${ }^{4}$ The use of newer chemotherapeutic regimens in clinical
Correspondence: Catalin Mihalcioiu Department of Medicine, McGill University Health Center/Royal Victoria Hospital, 687 Pine Avenue West, Montreal, QC, H3A IAI, Canada Fax + I 514843 I4I7 Email catalin.mihalcioiu@muhc.mcgill.ca 
practice has only modestly impacted survival outcomes. Although several therapeutic options are available for $\mathrm{MBC}$, including endocrine treatment, chemotherapy, radiotherapy, biologic treatment, alone or in combination, the preferred treatment consists of anthracycline-containing and/or taxanecontaining regimens. However, increasingly more patients are receiving anthracycline-containing and/or taxane-containing regimens as adjuvant therapy, restricting this option in the metastatic setting. There is currently no established standard of care for $\mathrm{MBC}$ and the prognosis remains poor. ${ }^{4}$ Various monotherapy and combination regimens have been evaluated in $\mathrm{MBC}$ patients, including capecitabine.

Capecitabine (Xeloda ${ }^{\circledR}$ ), is an orally bioavailable fluoropyrimidine carbamate approved in Canada for advanced $\mathrm{BC}$ or $\mathrm{MBC}$ as a monotherapy or combination therapy with lapatinib after failure of a prior anthracycline/taxane regimen. ${ }^{5}$ Capecitabine is converted to an active antineoplastic agent preferentially in tumor cells through a process requiring the thymidine phosphorylase (TP) enzyme, reason for which it has been postulated that TP expression might be an important determinant of efficacy and clinical outcome of fluoropyrimidine therapy. ${ }^{6-9}$ Capecitabine chemotherapy represents an attractive alternative to standard 5-fluorouracil-based therapy since it is given orally, has a better safety profile, and increases efficacy through selective activation at the tumor site. Although the TP enzyme is detected in both healthy and tumor tissue, its intratumoral expression has been reported to be three-fold to ten-fold higher, allowing for selective activation of capecitabine within tumor cells. The TP enzyme is expressed in tumor cells and in tumorassociated stromal cells. ${ }^{10}$ Expression of TP by stromal cells (ie, fibroblasts, macrophages, and lymphocytes) was found to be implicated in tumor neovascularization. TP expression has been shown to be highly variable among the different types of cancer. ${ }^{11}$ As depicted in Figure 1, the conversion of capecitabine to 5-fluorouracil is mediated by several enzymes, including hepatic carboxylesterase, cytidine deaminase, and TP. ${ }^{12}$

The TP enzyme plays an integral role in tumor angiogenesis, tumorigenicity, metastasis, and invasion. ${ }^{12-15} \mathrm{TP}$ is upregulated in both invasive lobular and ductal carcinoma (ILC/IDC). ${ }^{16,17}$ Moreover, TP expression has also been implicated in the development and progression of invasive BC. ${ }^{15}$ The presence and overexpression of TP in ductal carcinoma in situ (DCIS) has been reported. However, there was no significant correlation between TP expression and disease recurrence following treatment. ${ }^{18}$ Although one may expect an increase in TP expression in both IDC and DCIS, clinical studies reveal conflicting results. ${ }^{18-21}$

TP expression in tumor tissue is regulated by the tumor microenvironment (hypoxia, acidosis) which correlates significantly with microvessel density and a poor prognosis. ${ }^{22}$ TP is upregulated by stress such as hypoxia, hypoglycemia, radiation, and chemotherapeutic damage. ${ }^{23}$ Chemotherapeutic agents such as anthracycline, taxane, cyclophosphamide, and platinum compounds have been shown to upregulate TP. ${ }^{24}$

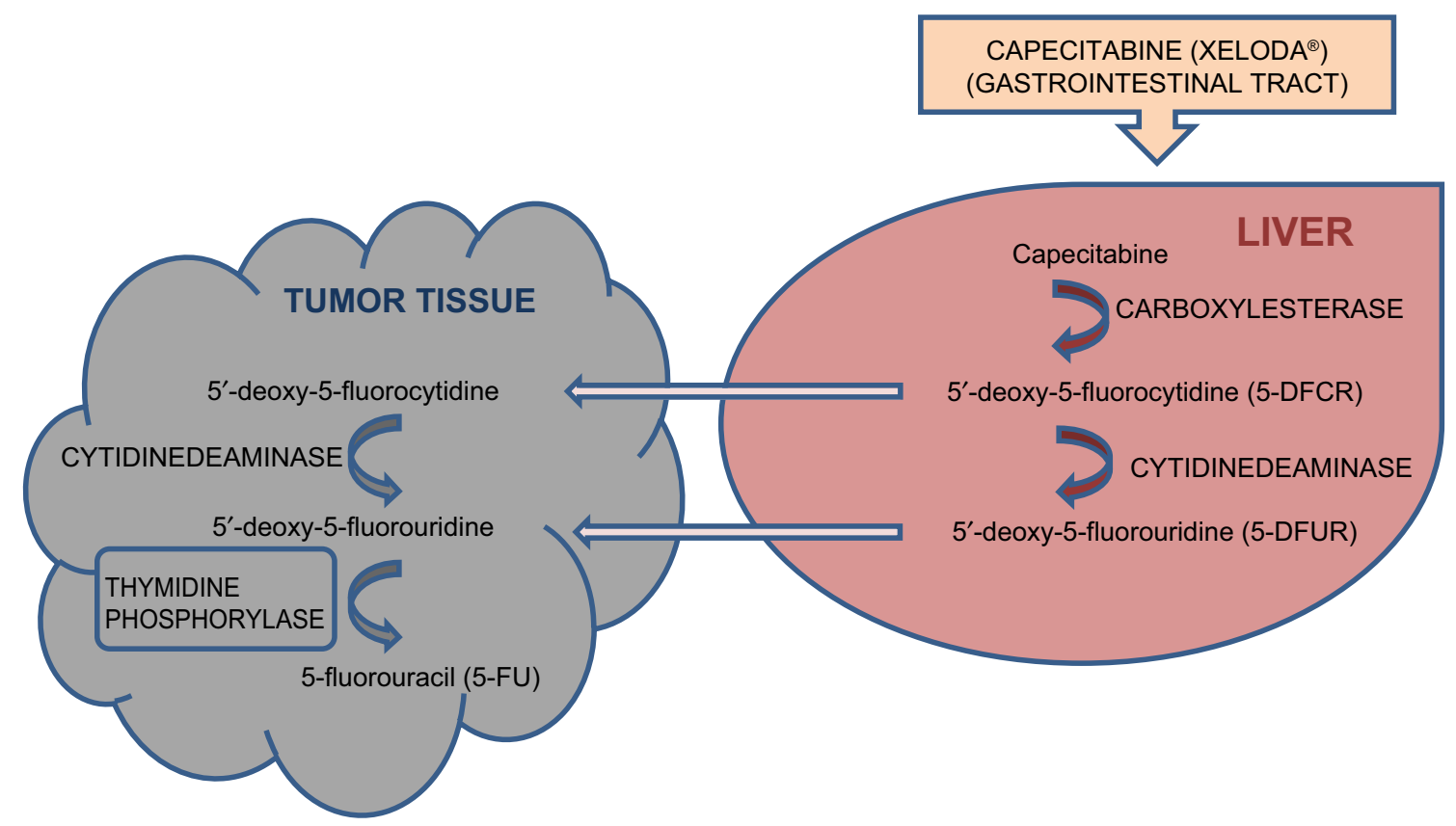

Figure I Metabolic conversion of the prodrug capecitabine to the active antimetabolite moiety, 5-fluorouracil. 
We and others have shown that inhibition of the epidermal growth factor receptor with the clinical drug gefitinib leads to an increase in TP levels in BC cells. ${ }^{25-27}$ It has also been demonstrated that high tumoral TP activity may serve as an indicator of response to fluoropyrimidine therapies. ${ }^{28}$ Due to the contradictory role of TP reported in the literature, targeting TP as a rationale for anticancer therapy or establishing its use as a biomarker remains unclear. Inhibitors of the TP enzyme are being synthesized as an attempt to fight invasion and angiogenesis. Promising novel therapeutic strategies being implemented in the adjuvant/neoadjuvant and metastatic setting induce an increase in intratumoral TP. As a result, it is difficult to predict the role of TP in patients exposed to fluoropyrimidine therapies. ${ }^{29}$ Although in several types of cancer, such as head and neck cancer, ${ }^{30}$ colorectal cancer, ${ }^{31}$ and advanced gastric cancers, ${ }^{32,33}$ tumoral TP expression has been shown to predict response to capecitabine therapy, most studies in advanced BC examining the predictive value of TP to capecitabine therapy have produced mixed results. ${ }^{21,34-37}$ The purpose of this retrospective pilot study was to assess if tumoral TP expression in both the invasive and in situ components in 18 patients with locally advanced or MBC correlates with therapeutic benefit from a capecitabine-based chemotherapy. These data will help us to establish the role of tumoral TP expression as a predictive biomarker for the response to capecitabine in patients with locally advanced $\mathrm{BC}$ or $\mathrm{MBC}$.

\section{Patients and methods Patient eligibility}

This retrospective pilot study was conducted at the McGill University Health Center, Department of Medicine, Division of Medical Oncology. The protocol was approved by the University Research Institute scientific review committee and Biomedical A Research ethics board. All patients who participated in the study provided written informed consent. The data were retrospectively selected based on the following criteria: locally advanced BC (stage III) or MBC (stage IV), treatment with a capecitabine-based chemotherapeutic regimen and completion of at least one cycle of capecitabine chemotherapy, and had a prior history of treatment with anthracycline or taxane therapy.

\section{Treatment regimens}

Patients received a capecitabine-based chemotherapy regimen when they progressed from prior therapies. Capecitabine was given orally at a dose of $1,000 \mathrm{mg} / \mathrm{m}^{2}$ twice daily for 2 weeks with a 1 -week rest period. Treatment was discontinued due to disease progression or unacceptable toxicity, adverse events, or patient/physician's decision.

\section{Tumor response evaluation}

The outcome of capecitabine therapy was evaluated by determining time to progression (TTP, assessed clinically or by imaging), defined as the time between starting therapy with capecitabine and disease progression or discontinuation of therapy by patient due to adverse events.

\section{Immunohistochemical evaluation of TP and $\mathrm{Ki} 67$}

Most of the histologic information used in the analysis was directly retrieved from the original pathology reports. The immunohistochemistry for estrogen receptor (ER) and progesterone receptor (PR) status, human epidermal growth factor receptor-2 (HER2) status, proliferating (Ki67) activity, and TP expression was carried out on formalin-fixed, paraffin-embedded samples of the primary excised tumor or, if not available, of the metastatic lesions. Immunostaining of the prognostic markers was accomplished using the following primary antibodies: ER status was tested using the Ventana Confirm anti-ER (SP1) rabbit monoclonal antibody; PR status was tested using the Ventana Confirm anti-PR (1E2) rabbit monoclonal antibody; HER2 status was tested using the Ventana Pathway anti-HER2/neu (4B5) rabbit monoclonal antibody; Ki67 labeling index was tested using the Ventana Confirm anti-Ki67 (30-9) rabbit monoclonal antibody; and TP expression was tested using the Novocastra ${ }^{\mathrm{TM}}$ lyophilized mouse monoclonal antibody platelet-derived endothelial growth factor (Leica Biosystems, Nussloch, Germany). Staining was performed using a Ventana XT automated (Ventana Medical Systems Inc., Roche, Tuscon, AZ, USA) slide stainer.

The ER and PR results were reported as the proportion of positively stained tumor cells, ranging from $0 \%$ to $100 \%$. ER and PR were positive when $\geq 1 \%$ of tumor cells showed positive nuclear staining. ${ }^{38}$

The HER2 expression level was scored based on the membrane staining intensity and percentage of positive cells on a scale of 0 to $3+$ in accordance with the guidelines published by Sauter et al. ${ }^{39}$ A negative HER2 included a score of 0 and +1 . A positive HER2 included a score of +3 . For a score of +2 to be considered positive, a fluorescent in situ hybridization analysis was required to show a ratio of more than 2.2. ${ }^{40}$

TP expression was analyzed by immunohistochemistry in tumor tissue (14 primary excised lesions, one needle biopsy 
tissue, and three metastatic lesions) and reported as a mixed nuclear/cytoplasmic ratio. For primary breast lesions, TP staining intensities were determined for both the invasive (ILC/IDC) and in situ (lobular carcinoma in situ/DCIS) components. Stromal TP intensities were not evaluated. Expression of the TP protein was correlated with BC type, ER and PR status, HER2 status, Ki67, and TTP. Staining intensities of TP in carcinoma cells was evaluated by three different pathologists, blinded to the tumor clinicopathologic data. The intensity of cytoplasmic and nuclear staining was categorized into $0,1,2$, or 3 , denoting 0 (no), +1 (weak), +2 (moderate), and +3 (strong) staining, respectively. The staining of normal mammary ductal epithelial cells was used as the internal control for moderate intensity as depicted in Figure 2A. The TP expression level for each case was defined as positive if the predominant intensity was 2 or 3 , and as negative if the predominant intensity was 0 or $1 .{ }^{41}$ At least $10 \%$ of tumor cells had to be +2 or +3 in order to be defined as positive. As the material comprised a mixture of excision and biopsy material, a quantitative measurement was not performed. Representative images of moderate, weak, and no TP staining in DCIS and invasive carcinoma are depicted in Figure 2B-G.

The Ki67 score was defined as the percentage of the total number of 200 tumor cells with nuclear immunohistochemical staining positive for Ki67. In sections stained for Ki67, the area with the highest fraction of Ki67-stained cells was chosen using a $10 \times$ objective magnification. A $40 \times$ objective was used to count arbitrarily the fraction of Ki67+-positive

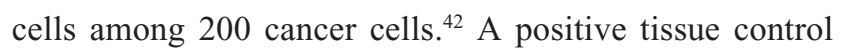
was run with every staining procedure performed. The evaluations were made by a pathologist. Ki67 expression was scored as low and high, respectively, if $\leq 14 \%$ and $>14 \%$ of the tumor cells showed positive nuclear staining for Ki67 (Figure 2H). ${ }^{43}$

\section{Statistical analysis}

All analyses were performed using GraphPad software (GraphPad Software Inc., San Diego, CA, USA). The significance of differences in the means was determined by an unpaired, two-tailed $t$-test. Means were considered to be statistically significant when $P$-values were less than 0.05 .

\section{Results}

Eighteen female patients were included in this study, with a median age of 56 (range 41-83) years. All patients received capecitabine, and the characteristics of the 18 patients are presented in Table 1. The majority of pathologic specimens used for TP staining were surgical biopsies. Three pathologic specimens were metastatic lesions, two were liver core biopsies, and the third was a chest wall tumor excision; immunohistochemistry was done on tumor cells from all three patients. The TP score was 0 for these three patients. Of the two patients with liver biopsies, one had a long survival and is still alive, and the other had a short TTP of 6 months. Bioactivation of capecitabine is not affected in patients with hepatic insufficiency. The third patient had an excisional biopsy from the chest wall, with a TP score of 0 , a lobular cancer subtype, and had a long TTP.

All but three patients had IDC, and all but one were stage IV. Of the 18 patients, eleven did not have lobular carcinoma in situ or DCIS. Of the seven patients with DCIS, five were TP-positive (grade 2) and two were TP-negative (grade 0 and 1). Of the 18 patients, only two stained positive for TP (grade 2) on the invasive tumor (IDC). Both these patients were ER-negative. None of the patients were grade 3 . There was no correlation between TP positivity and Ki67 expression, but all HER2-positive patients had high Ki67.

Of the 15 patients with IDC, six were ER-negative and nine were ER-positive. Four patients had tumors with low Ki67 expression $(\leq 14 \%)$ whereas the other 14 patients had high Ki67 (>14\%). Among the 18 patients treated with capecitabine, four had to discontinue due to intolerance. The most common reported adverse events attributable to capecitabine chemotherapy were gastrointestinal (ie, diarrhea, nausea/vomiting) and dermatologic (ie, palmarplantar erythrodysesthesia).

None of the three patients with ILC had positive TP staining, and two had high and one had low Ki67 expression. All were ER-positive, PR-positive, and HER2-negative (Table 1). Their TTP ranged from 6 to more than 33 months (Table 2).

Of the six patients with ER-negative tumors (Table 2), four expressed TP-negative staining (two grade 0 and two grade +1$)$ and two stained positive for TP $($ grade +2$)$. Five of the six patients expressed high levels of Ki67 (>14\%). The DCIS component was present in three of the six patients, with two patients expressing moderate staining for TP in both IDC and correspondent DCIS sample. TTP in the six ER-negative patients ranged between 2 and 15 months, with a median TTP of 7.5 months.

In correlating TP expression with ER, PR, and HER2 status, our data showed that four patients had triple-negative breast cancer (TNBC). Two of these patients were positive for TP (grade +2 ) and two had some TP activity (grade +1 ). The two patients with ER/PR-negative/HER2-positive tumors were negative for TP (grade 0 ). 

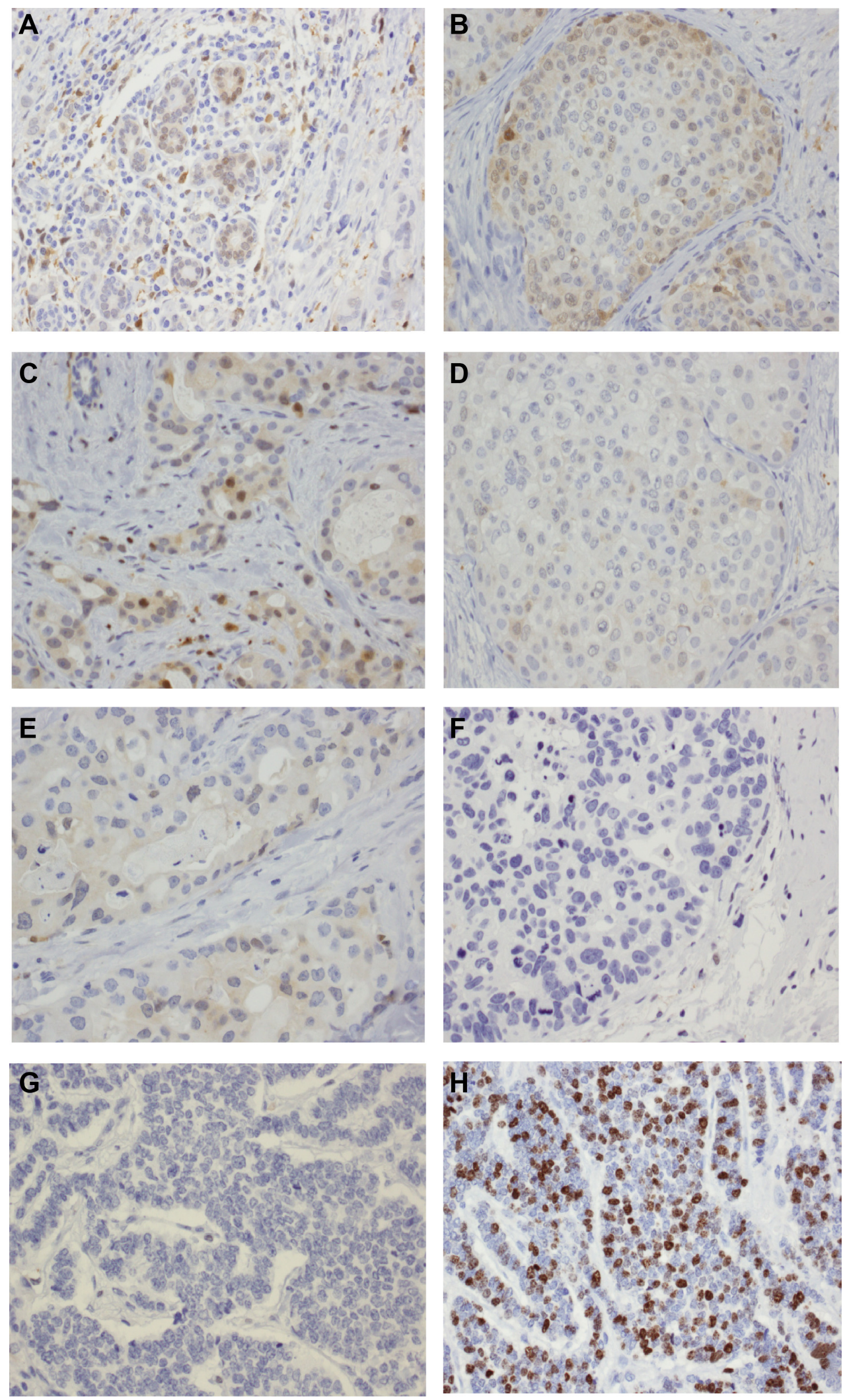

Figure 2 Immunohistochemistry showing TP expression in carcinoma of the breast (magnification, 400x). Representative images of immunohistochemistry staining patterns for mixed nuclear/cytoplasmic TP protein in normal epithelium and in primary or metastatic lesions.

Notes: (A) Normal mammary ductal epithelium showing moderate staining $(+2)$ of TP, $(\mathbf{B})$ DCIS showing moderate staining $(+2)$ of TP, $(\mathbf{C})$ invasive carcinoma showing moderate staining (+2) of TP, (D) DCIS showing weak staining (+I) of TP, (E) invasive carcinoma showing weak staining $(+I)$ of TP, (F) DCIS showing no staining $(0)$ of TP, (G) invasive carcinoma showing no staining $(0)$ of TP, and $(\mathbf{H})$ nuclear staining of Ki67.

Abbreviations: DCIS, ductal carcinoma in situ; TP, thymidine phosphorylase. 
Table I Patient tumor characteristics

\begin{tabular}{|c|c|c|c|c|c|c|c|c|c|c|}
\hline \multirow[t]{2}{*}{ ID } & \multirow[t]{2}{*}{$\begin{array}{l}\text { Age at diagnosis, } \\
\text { years }\end{array}$} & \multirow[t]{2}{*}{$\begin{array}{l}\text { Type } \\
\text { of biopsy }\end{array}$} & \multirow[t]{2}{*}{$\begin{array}{l}\text { Type } \\
\text { of BC }\end{array}$} & \multirow[t]{2}{*}{ Stage } & \multicolumn{2}{|c|}{$\begin{array}{l}\text { TP expression in } \\
\text { DCIS and ILC/IDC }\end{array}$} & \multirow[t]{2}{*}{ Ki67 } & \multicolumn{3}{|c|}{ Receptor status } \\
\hline & & & & & DCIS & ILC/IDC & & ER & PR & HER2 \\
\hline I & 70 & Surgical & IDC & IV & +2 & +2 & $13 \%$ & - & - & - \\
\hline 2 & 55 & Surgical & IDC & IV & Not present & +1 & $59 \%$ & - & - & - \\
\hline 3 & 51 & Surgical & IDC & IV & Not present & +1 & $26 \%$ & - & - & - \\
\hline 4 & 51 & Surgical & IDC & IV & 0 & 0 & $60 \%$ & - & - & - \\
\hline 5 & 56 & Surgical & IDC & IV & Not present & 0 & $24 \%$ & - & - & + \\
\hline 6 & 45 & Surgical & IDC & IV & +2 & +2 & $26.5 \%$ & - & - & + \\
\hline 7 & 49 & Surgical & IDC & IV & +2 & +1 & $25 \%$ & + & + & $\mathrm{N} / \mathrm{A}$ \\
\hline 8 & 72 & Surgical & IDC & IV & Not present & 0 & $22.5 \%$ & + & - & - \\
\hline 9 & 65 & Surgical & IDC & IV & Not present & 0 & $50 \%$ & + & + & + \\
\hline 10 & 48 & Metastatic lesion & ILC & IV & Not present & 0 & $26 \%$ & + & + & - \\
\hline II & 62 & Metastatic lesion & IDC & IV & Not present & 0 & $15 \%$ & + & + & + \\
\hline 12 & 54 & Surgical & IDC & IV & +2 & 0 & $33.5 \%$ & + & + & - \\
\hline 13 & 43 & Metastatic lesion & ILC & IV & Not present & 0 & $20 \%$ & + & + & - \\
\hline 14 & 59 & Surgical & ILC & IV & Not present & 0 & $1 \%$ & + & + & - \\
\hline 15 & 56 & Surgical & IDC & IV & +1 & 0 & $14 \%$ & + & + & - \\
\hline 16 & 83 & Surgical & IDC & IV & Not present & +1 & $30.5 \%$ & + & - & - \\
\hline 17 & 65 & Surgical & IDC & IV & +2 & +1 & $37.5 \%$ & + & - & + \\
\hline 18 & 41 & Needle & IDC & III & Not present & 0 & $37.5 \%$ & + & + & + \\
\hline
\end{tabular}

Abbreviations: BC, breast cancer; DCIS, ductal carcinoma in situ; ER, estrogen receptor; PR, progesterone receptor; ILC, invasive lobular carcinoma; IDC, invasive ductal carcinoma; TP, thymidine phosphorylase; N/A, not available.

Table 2 Relationship between patient ER-positive/negative receptor status and tumoral TP expression, and the clinical benefit to a capecitabine-based chemotherapeutic regimen measured as TTP

\begin{tabular}{|c|c|c|c|c|c|c|c|c|}
\hline \multirow[t]{2}{*}{ ID } & \multirow[t]{2}{*}{$\begin{array}{l}\text { Stage and type } \\
\text { of } B C\end{array}$} & \multicolumn{2}{|c|}{$\begin{array}{l}\text { TP expression in DCIS } \\
\text { and ILC/IDC }\end{array}$} & \multirow[t]{2}{*}{ Ki67 } & \multicolumn{3}{|c|}{ Receptor status } & \multirow[t]{2}{*}{ TTP on capecitabine-based therapy } \\
\hline & & DCIS' $^{\prime}$ & ILC/IDC $^{2}$ & & ER & PR & HER2 & \\
\hline \multicolumn{9}{|c|}{ ER-negative group } \\
\hline I & Stage IV; IDC & +2 & +2 & $13 \%$ & - & - & - & 9 months \\
\hline 2 & Stage IV; IDC & Not present & +1 & $59 \%$ & - & - & - & 15 months \\
\hline 3 & Stage IV; IDC & Not present & +1 & $26 \%$ & - & - & - & 5 months \\
\hline 4 & Stage IV; IDC & 0 & 0 & $60 \%$ & - & - & - & 2 months \\
\hline 5 & Stage IV; IDC & Not present & 0 & $24 \%$ & - & - & + & 6 months \\
\hline 6 & Stage IV; IDC & +2 & +2 & $26.5 \%$ & - & - & + & 9 months \\
\hline \multicolumn{9}{|c|}{ ER-positive group } \\
\hline 7 & Stage IV; IDC & +2 & +1 & $25 \%$ & + & + & N/A & 19 months \\
\hline 8 & Stage IV; IDC & Not present & 0 & $22.5 \%$ & + & - & - & 2 months \\
\hline 9 & Stage IV; IDC & Not present & 0 & $50 \%$ & + & + & + & 4 months \\
\hline 10 & Stage IV; ILC & Not present & 0 & $26 \%$ & + & + & - & 33 months \\
\hline II & Stage IV; IDC & Not present & 0 & $15 \%$ & + & + & + & 30 months \\
\hline 12 & Stage IV; IDC & +2 & 0 & $33.5 \%$ & + & + & - & 7 months \\
\hline 13 & Stage IV; ILC & Not present & 0 & $20 \%$ & + & + & - & 6 months \\
\hline 14 & Stage IV; ILC & Not present & 0 & $1 \%$ & + & + & - & 36 months and still on it \\
\hline \multicolumn{9}{|c|}{ Patients who discontinued due to adverse events } \\
\hline 15 & Stage IV; IDC & +1 & 0 & $14 \%$ & + & + & - & Dizzy; $\sim 12$ days \\
\hline 16 & Stage IV; IDC & Not present & +1 & $30.5 \%$ & + & - & - & $\begin{array}{l}\text { Developed colitis secondary to capecitabine, } \\
\text { hand/foot syndrome, bloody diarrhea; } \sim 2 \text { months }\end{array}$ \\
\hline 17 & Stage IV; IDC & +2 & +1 & $37.5 \%$ & + & - & + & Recurrent diarrhea; $\sim 3$ months \\
\hline 18 & $\begin{array}{l}\text { Locally advanced } \\
\text { stage III; IDC }\end{array}$ & Not present & 0 & $37.5 \%$ & + & + & + & $\begin{array}{l}\text { Poor tolerance to capecitabine, flare-up of } \\
\text { Crohn's disease and blurred vision; I month }\end{array}$ \\
\hline
\end{tabular}

Notes: ${ }^{1,2}$ TP intensity of cytoplasmic and nuclear staining was categorized into 0, I, 2, or 3, denoting 0 (no), I (weak), 2 (moderate), and 3 (strong) staining, respectively. The LCIS component was not present in any of the specimens.

Abbreviations: BC, breast cancer; DCIS, ductal carcinoma in situ; ER, estrogen receptor; PR, progesterone receptor; ILC, invasive lobular carcinoma; IDC, invasive ductal carcinoma; TTP, time to progression; TP, thymidine phosphorylase; N/A, not available. 
The ER-positive tumor group (Table 2) included a total of 12 patients, of whom four discontinued due to intolerance of capecitabine and all had negative TP staining in the invasive component. Two of the eight patients who tolerated capecitabine had DCIS present and stained positive for TP (grade 2). All eight patients who tolerated capecitabine were negative for TP staining. Two of the eight patients were HER2-positive and ER/PR-positive. Seven of the eight patients had a high Ki67 proliferative index. The TTP of these eight patients ranged from 2 to 36 months, with a median TTP of 13 months.

In the ER-positive group, two patients were ER-positive/ HER2-positive and five were ER-positive/HER2-negative. All seven patients were TP-negative (grade 0). Only one ER-positive patient did not have HER2-status reported and is the only patient with some TP staining (grade +1 ).

Out of the 14 ER-positive/negative patients who did not discontinue treatment due to side effects, none had lobular carcinoma in situ and five had a DCIS component present. Four of the five DCIS patients were TP-positive (grade 2) and all had a TTP ranging from 7 to 19 months compared with the one DCIS-TP-negative patient who had a TTP of 2 months. The two IDC patients positive for TP $($ grade +2$)$ were also TP-positive in DCIS (grade +2 ) and both had a TTP of 9 months.

Overall, for these 18 patients, there was no significant correlation between TP positivity and response to capecitabine as measured by TTP. The two patients with a TTP longer than 30 months were both TP-negative, ER/PR-positive, HER2negative, had a low Ki67, and displayed an ILC subtype. The ER-negative group had a more significant correlation with TP positivity (Table 2) but this did not translate into therapeutic benefit.

\section{Discussion}

The era of personalized medicine is escalating due to the progress made in understanding tumor biology and molecular phenotypes in BC. This has led to identification of new biomarkers that allow patients to receive personalized treatments. Apart from tumor size, axillary lymph node status, tumor grade Ki67, and ER, PR, and HER2 status, there are no other biomarkers predictive of the response to chemotherapy. ${ }^{4-47}$

The TP enzyme has been extensively investigated in order to determine its role as a biomarker in predicting the response to capecitabine therapy in patients with locally advanced BC or MBC. In our study, among all the variables considered, a correlation was observed between ER-positive/negative tumors and TP status. We observed a significant correlation of TP positivity in the invasive component of ER-negative tumors when compared with ER-positive tumors $(P<0.05)$. This is corroborated by other investigators who also found that TP expression was significantly higher in ER-negative tumors. ${ }^{16,17,48}$ The DCIS component in both ER-positive/negative patients correlated with TP positivity; however, a statistically significant correlation could not be established since it was not present in many patients in our population. In order for TP to be a useful predictive biomarker, its positivity in tumor tissue has to correlate with TTP. In this study, we found no correspondence between TP positivity in the invasive tumor and TTP in the group of capecitabine-treated patients. However, ER-positive/negative patients staining positive for TP in the DCIS component had better TTP (7-19 months) when compared with the one TP-negative patient with DCIS who had a TTP of 2 months. Given the critical role of the TP enzyme in the activation of capecitabine, one would expect patients with stronger staining for TP to respond better to treatment with capecitabine.

Our data, however, are consistent with the literature, since clinical studies designed to assess the levels of TP in DCIS and IDC have yielded mixed results. ${ }^{15-18}$ Some studies suggest that TP has angiogenic effects in breast carcinoma whereas others indicate that TP has a minimal role in tumor angiogenesis. ${ }^{7,15,16,18,20,21}$ In addition, in a study conducted by Erkus et al ${ }^{19}$ TP staining in both DCIS and IDC did not correlate with the conventional prognostic markers, ie, age, tumor diameter, lymph node, ER, PR, HER2, P53, and Ki67 status, and tumor grade. Likewise, in our study, we did not see an association between TP staining intensities and Ki67.

The median TTP in patients with ER-positive and ER-negative tumors was estimated to be 13 months and 7.5 months, respectively, indicating a trend for patients with ER-positive tumors to have a longer TTP than those with ER-negative tumors $(P=0.14)$. Andreetta et $\mathrm{al}^{49}$ also reported a statistically significant association between TTP and ER positivity, where ER-positive tumors had significantly longer TTP when compared with ER-negative tumors. Our data did suggest that patients in the ER-positive group were TP-negative $(P<0.05)$, although we could not correlate their TP status and TTP. The better outcome observed in this group could not have been influenced by a capecitabine-TP interaction. TP induces angiogenesis, and ER-negative tumors are associated with increased angiogenesis, which may explain the higher TP levels expressed in these tumors and poor outcome of therapy. 
When TP expression was correlated in TNBC and HER2-positive tumors, our results show that triple-negative tumors had more TP activity when compared with HER2positive tumors. Patients with TNBC had a TTP ranging from 5 to 15 months, with a median TTP of 9 months when compared with HER2-positive patients who had a TTP ranging from 2 to 6 months with a median TTP of 4 months. This result suggests that patients with TNBC might benefit from capecitabine chemotherapy more than HER2-positive patients. When considering the poor prognosis and lack of benefit of targeted therapies in TNBC patients, capecitabine would be a good therapeutic choice for TP-positive patients. In retrospect, we should have collected a larger TP-positive/ negative capecitabine-treated sample and analyze for TTP in ER-positive, ER-negative, HER2-positive, HER2-negative, and TNBC patients.

In order to have a more meaningful correlation between TP positivity and disease control on capecitabine, intratumoral dihydropyrimidine dehydrogenase (DPD) should have been evaluated as well. The DPD enzyme is involved in the catabolism of fluoropyrimidines, and an elevated tumor DPD level would result in a low TP/DPD ratio. According to the literature, high intratumoral DPD levels confer resistance to treatment, whereas its cytotoxic efficacy is improved in tumor cells expressing a high TP/DPD ratio. ${ }^{50-53}$ Further, the statistical significance of this work was affected by the small sample size due to the limited number of patients receiving capecitabine who were eligible for our retrospective singlecenter study, and the current observed trends are worth further investigation in a larger study population.

\section{Conclusion}

In this retrospective pilot study in advanced $\mathrm{BC}, \mathrm{TP}$ expression in tumor tissue did not correlate with response to capecitabinebased chemotherapy. Our ER-negative patients had a higher associated positivity for TP staining and for Ki67, but their TP status did not correlate with a predictive TTP. By contrast, there was no correlation between ER-positivity and TP positivity. Consequently, the relationship between TP and ER status requires further scientific and clinical investigation.

\section{Acknowledgments}

MAT thanks Fonds de Recherche du Québec-Santé for a doctoral award. BJC is grateful to the Canadian Institutes of Health Research for financial support (Grant \#: MOP-89968).

\section{Disclosure}

The authors report no conflicts of interest in this work.

\section{References}

1. Canadian Cancer Society. Breast cancer statistics. Available from: http://www.cancer.ca/en/cancer-information/cancer-type/breast/ statistics/?region=nb. Accessed November 21, 2012.

2. Chung CT, Carlson RW. Goals and objectives in the management of metastatic breast cancer. Oncologist. 2003;8:514-520.

3. Early Breast Cancer Trialists' Collaborative Group. Effects of chemotherapy and hormonal therapy for early breast cancer on recurrence and 15-year survival: an overview of the randomised trials. Lancet. 2005; 365:1687-1717.

4. Rivera E. Management of metastatic breast cancer monotherapy options for patients resistant to anthracyclines and taxanes. Am J Clin Oncol. 2010; 33:176-185.

5. Capecitabine $\left(\mathrm{Xeloda}^{\circledR}\right)$ product monograph. Available from: http:// webprod5.hc-sc.gc.ca/dpd-bdpp/info.do? code=61873\&lang=eng. Accessed November 21, 2014.

6. Aprile G, Mazzer M, Moroso S, Puglisi F. Pharmacology and therapeutic efficacy of capecitabine: focus on breast and colorectal cancer. Anticancer Drugs. 2009;20:217-229.

7. Toi M, Atiqur Rahman M, Bando H, Chow LW. Thymidine phosphorylase (platelet-derived endothelial-cell growth factor) in cancer biology and treatment. Lancet Oncol. 2005;6:158-166.

8. Schüller J, Cassidy J, Dumont E, et al. Preferential activation of capecitabine in tumor following oral administration to colorectal cancer patients. Cancer Chemother Pharmacol. 2000;45:291-297.

9. Lee SJ, Choi YL, Park YH, et al. Thymidylate synthase and thymidine phosphorylase as predictive markers of capecitabine monotherapy in patients with anthracycline- and taxane-pretreated metastatic breast cancer. Cancer Chemother Pharmacol. 2011;68:743-751.

10. Budman DR, Meropol NJ, Reigner B, et al. Preliminary studies of a novel oral fluoropyrimidine carbamate: capecitabine. J Clin Oncol. 1998; 16:1795-1802.

11. Kamoshida S, Shiogama K, Shimomura R, et al. Immunohistochemical demonstration of fluoropyrimidine-metabolizing enzymes in various types of cancer. Oncol Rep. 2005;14:1223-1230.

12. Quidde J, Arnold D, Stein A. Clinical management of localized colon cancer with capecitabine. Clin Med Insights Oncol. 2012;6:363-373.

13. El Omari K, Bronckaers A, Liekens S, Pérez-Pérez MJ, Balzarini J, Stammers DK. Structural basis for non-competitive product inhibition in human thymidine phosphorylase: implications for drug design. Biochem J. 2006;399:199-204.

14. Yu EJ, Lee Y, Rha SY, et al. Angiogenic factor thymidime phosphorylase increases cancer cell invasion activity in patients with gastric adenocarcinoma. Mol Cancer Res. 2008;6:1554-1566.

15. Teo NB, Shoker BS, Jarvis C, Sloane JP, Holcombe C. Thymidine phosphorylase expression and stromal vascularity in ductal carcinoma in situ of the breast. J Clin Pathol. 2003;56:919-923.

16. Nagaoka H, Iino Y, Takei H, Morishita Y. Platelet-derived endothelial cell growth factor/thymidine phosphorylase expression in macrophages correlates with tumour angiogenesis and prognosis in invasive breast cancer. Int J Oncol. 1998;13:449-454.

17. Yonenaga F, Takasaki T, Ohi Y, et al. The expression of thymidine phosphorylase/platelet-derived endothelial cell growth factor is correlated to angiogenesis in breast cancer. Pathol Int. 1998;48:850-856.

18. Engels K, Fox SB, Whitehouse RM, Gatter KC, Harris AL. Upregulation of thymidine phosphorylase expression is associated with a discrete pattern of angiogenesis in ductal carcinoma in situ of the breast. J Pathol. 1997;182:414-420.

19. Erkus M, Meteoglu I, Culhaci N, Meydan N, Erdogdu I. Does thymidine phosphorylase correlate with angiogenesis in intraductal cancinoma of the breast? Saudi Med J. 2006;27:1329-1333.

20. Lee AH, Dublin EA, Bobrow LG. Angiogenesis and expression of thymidine phosphorylase by inflammatory and carcinoma cells in ductal carcinoma in situ of the breast. J Pathol. 1999;187:285-290.

21. Yang Q, Barbareschi M, Mori I, et al. Prognostic value of thymidine phosphorylase expression in breast carcinoma. Int J Cancer. 2002; 97:512-517. 
22. Nakajima Y, Madhyastha R, Maruyama M. 2-Deoxy-D-ribose, a downstream mediator of thymidine phosphorylase, regulates tumor angiogenesis and progression. Anticancer Agents Med Chem. 2009;9:239-245.

23. Toi M, Bando H, Horiguchi S, et al. Modulation of thymidine phosphorylase by neoadjuvant chemotherapy in primary breast cancer. Br J Cancer. 2004;90:2338-2343.

24. Twelves C. Vision of the future: capecitabine. Oncologist. 2001;6 Suppl4: 35-39.

25. Magné N, Fischel JL, Dubreuil A, et al. ZD1839 (Iressa) modifies the activity of key enzymes linked to fluoropyrimidine activity rational basis for a new combination therapy with capecitabine. Clin Cancer Res. 2003;9:4735-4742.

26. Ait-Tihyaty M, Rachid Z, Mihalcioiu C, Jean-Claude BJ. Inhibition of EGFR phosphorylation in a panel of human breast cancer cells correlates with synergistic interactions between gefitinib and 5'-DFUR, the bioactive metabolite of Xeloda ${ }^{\circledR}$. Breast Cancer Res Treat. 2012; 133:217-226.

27. Ait-Tihyaty M, Rachid Z, Larroque-Lombard A-L, Jean-Claude BJ. ZRX1, the first EGFR inhibitor-capecitabine based combi-molecule, requires carboxylesterase-mediated hydrolysis for optimal activity. Invest New Drugs. 2013;31:1409-1423.

28. Bronckaers A, Gago F, Balzarini J, Liekens S. The dual role of thymidine phosphorylase in cancer development and chemotherapy. Med Res Rev. 2009;29:903-953.

29. Ciccolini J, Evrard A, Cuq P. Thymidine phosphorylase and fluoropyrimidines efficacy: a Jekyll and Hyde story. Curr Med Chem Anticancer Agents. 2004;4:71-81.

30. Saito K, Khan K, Yu SZ, et al. The predictive and therapeutic value of thymidine phosphorylase and dihydropyrimidine dehydrogenase in capecitabine (Xeloda)-based chemotherapy for head and neck cancer. Laryngoscope. 2009;119:82-88.

31. Takebayashi Y, Akiyama S, Akiba S, et al. Clinicopathologic and prognostic significance of an human angiogenic factor, thymidine phosphorylase, in human colorectal carcinoma. J Natl Cancer Inst. 1996;88:1110-1117.

32. Gao J, Lu M, Yu JW, Li YY, Shen L. Thymidine phosphorylase/ $\beta$ tubulin III expressions predict the response in Chinese advanced gastric cancer patients receiving first-line capecitabine plus paclitaxel. BMC Cancer. 2011;11:177.

33. Lu M, Gao J, Wang XC, Shen L. Expressions of thymidylate synthase, thymidine phosphorylase, class III $\beta$-tubulin, and excision repair cross-complementing group 1 predict response in advanced gastric cancer patients receiving capecitabine plus paclitaxel or cisplatin. Chin J Cancer Res. 2011;23:288-294.

34. Morel AN, Mehta S, Taylor MA, et al. Relationship of capecitabine response to thymidine phosphorylase expression and overall survival in advanced breast cancer. Cancer Res. 2010;70:24 Suppl 2:P2-09-24.

35. Li H, Suo Z, Zhang Y, et al. The prognostic significance of thymidine phosphorylase, thymidylate synthase and dihydropyrimidine dehydrogenase mRNA expressions in breast carcinomas. Histol Histopathol. 2004; 19:129-136.

36. Tominaga T, Toi M, Abe O; 5'-BC Study Group. Prognostic and predictive value of thymidine phosphorylase activity in early-stage breast cancer patients. Clin Breast Cancer. 2002;3:55-64.

37. Puglisi F, Cardellino GG, Crivellari D, et al. Thymidine phosphorylase expression is associated with time to progression in patients receiving low-dose, docetaxel-modulated capecitabine for metastatic breast cancer. Ann Oncol. 2008;19:1541-1546.

OncoTargets and Therapy

\section{Publish your work in this journal}

OncoTargets and Therapy is an international, peer-reviewed, open access journal focusing on the pathological basis of all cancers, potential targets for therapy and treatment protocols employed to improve the management of cancer patients. The journal also focuses on the impact of management programs and new therapeutic agents and protocols on

Submit your manuscript here: http://www.dovepress.com/oncotargets-and-therapy-journal
38. Hammond ME, Hayes DF, Dowsett M, et al. American Society of Clinical Oncology/College of American pathologists guideline recommendations for immunohistochemical testing of estrogen and progesterone receptors in breast cancer. Arch Pathol Lab Med. 2010;134:e48-e72.

39. Sauter G, Lee J, Bartlett JM, Slamon DJ, Press MF. Guidelines for human epidermal growth factor receptor 2 testing: biologic and methodologic considerations. J Clin Oncol. 2009;27:1323-1333.

40. Wolff AC, Hammond ME, Schwartz JN, et al. American Society of Clinical Oncology/College of American Pathologists guideline recommendations for human epidermal growth factor receptor 2 testing in breast cancer. J Clin Oncol. 2007;25:118-145.

41. Tsuda H, Akiyama F, Kurosumi M, Sakamoto G. Reproducible immunohistochemical criteria based on multiple raters' judgements for expression of thymidine phosphorylase in breast cancer tissue. Breast Cancer Res Treat. 2004;86:215-223.

42. Jalava P, Kuopio T, Juntti-Patinen L, Kotkansalo T, Kronqvist P, Collan Y. Ki67 immunohistochemistry: a valuable marker in prognostication but with a risk of misclassification: proliferation subgroups formed based on Ki67 immunoreactivity and standardized mitotic index. Histopathology. 2006;48:674-682.

43. Cheang MC, Chia SK, Voduc D, et al. Ki67 index, HER2 status, and prognosis of patients with luminal B breast cancer. J Natl Cancer Inst. 2009;101:736-750.

44. Weigel MT, Dowsett M. Current and emerging biomarkers in breast cancer: prognosis and prediction. Endocr Relat Cancer. 2010;17: R245-R262.

45. Jonat W, Arnold N. Is the Ki67 labelling index ready for clinical use? Ann Oncol. 2011;22:500-502.

46. Dowsett M, Dunbier AK. Emerging biomarkers and new understanding of traditional markers in personalized therapy for breast cancer. Clin Cancer Res. 2008;14:8019-8026.

47. Fasching PA, Heusinger K, Haeberle L, et al. Ki67, chemotherapy response, and prognosis in breast cancer patients receiving neoadjuvant treatment. BMC Cancer. 2011;11:486.

48. Umemura S, Shirane M, Takekoshi S, Tokuda Y, Mori K, Osamura RY. High expression of thymidine phosphorylase in basal-like breast cancers: stromal expression in EGFR- and/or CK5/6-positive breast cancers. Oncol Lett. 2010;1:261-266.

49. Andreetta C, Puppin C, Minisini A, et al. Thymidine phosphorylase expression and benefit from capecitabine in patients with advanced breast cancer. Ann Oncol. 2009;20:265-271.

50. Beck A, Etienne MC, Chèradame S, et al. A role for dihydropyrimidine dehydrogenase and thymidylate synthase in tumour sensitivity to fluorouracil. Eur J Cancer. 1994;30A:1517-1522.

51. Etienne MC, Chèradame S, Fischel JL, et al. Response to fluorouracil therapy in cancer patients: the role of tumoral dihydropyrimidine dehydrogenase activity. J Clin Oncol. 1995;13:1663-1670.

52. Ishikawa T, Sekiguchi F, Fukase Y, Sawada N, Ishitsuka H. Positive correlation between the efficacy of capecitabine and doxifluridine and the ratio of thymidine phosphorylase to dihydropyrimidine dehydrogenase activities in tumors in human cancer xenografts. Cancer Res. 1998;58:685-690.

53. Takayama T, Mugiya S, Sugiyama T, et al. High levels of thymidine phosphorylase as an independent prognostic factor in renal cell carcinoma. Jpn J Clin Oncol. 2006;36:564-569.

\section{Dovepress}

patient perspectives such as quality of life, adherence and satisfaction. The manuscript management system is completely online and includes a very quick and fair peer-review system, which is all easy to use. Visit http://www.dovepress.com/testimonials.php to read real quotes from published authors. 